Relmecs, junio 2018, vol. 8, no. 1, e039, ISSN 1853-7863

Universidad Nacional de La Plata. Facultad de Humanidades y Ciencias de la Educación. Centro Interdisciplinario de Metodología de las Ciencias Sociales.

Red Latinoamericana de Metodología de las Ciencias Sociales

\title{
La pregunta por la reflexividad como práctica ineludible de la ciencia social
}

\author{
Reseña de: Juan Piovani y Leticia Muñiz Terra, coordinadores \\ (2018). ¿Condenados a la reflexividad? Apuntes para repensar el \\ proceso de investigación social. Buenos Aires: Biblos - CLACSO.
}

\author{
Santiago García Martín (*), Pedro Porta Fernández (**) \\ * Centro de Investigaciones Sociohistóricas - Instituto de Investigaciones en Humanidades y Ciencias Sociales - \\ Universidad Nacional de La Plata - CONICET, Argentina, ** Centro Interdisciplinario de Metodología de las \\ Ciencias Sociales - Instituto de Investigaciones en Humanidades y Ciencias Sociales - Universidad Nacional \\ de La Plata - CONICET, Argentina | sgarciamartin@fahce.unlp.edu.ar
}

El interrogante que titula el libro compilado por Juan Piovani y Leticia Muñiz Terra coloca la cuestión de la reflexividad metodológica en un sitio que nos invita a repensar nuestras propias prácticas de producción de conocimiento a la luz de una tarea más vasta, de un cometido que resulta a la vez sustancial e interminable: ser reflexivas y reflexivos constituye un compromiso con el desenvolvimiento teórico-metodológico y empírico de las ciencias sociales. Representa también, para este volumen, una apuesta a fortalecer la construcción de espacios de deliberación crítica que entrecruzan las fronteras porosas de campos como la sociología, la antropología y la metodología de un modo fecundo y creativo. ¿Condenados a la reflexividad? sugiere, quizá, que la suerte del estado actual de las disciplinas sociales y humanas -o su estatus frente a otros discursos y lógicas de la vida social que también dicen, conocen y confrontan sobre sus objetos- en parte corre detrás de dicha pregunta.

La respuesta por la reflexividad en el proceso de investigación social que ensaya este libro reúne a un conjunto de investigadoras e investigadores que ofrecen variadas respuestas sobre su acepción y uso, sobre su carácter multiescalar o sus diferentes niveles de análisis, y sobre la productividad de su empleo para diferentes enfoques metodológicos; todo ello sobre la base de experiencias de 
investigación empíricas concretas. Una primera parte del libro está dedicada a las discusiones de índole teórica que la sociología y la antropología social proponen para la noción de reflexividad metodológica. Luego, en una segunda parte, se adentra de lleno en el análisis de investigaciones empíricas para restituir el rol clave de la práctica reflexiva en los distintos momentos del proceso de investigación a partir de tres enfoques metodológicos: el enfoque biográfico, la etnografía y los métodos mixtos.

La compilación comienza con el Prefacio “Ciencia y reflexividad” a cargo del sociólogo británico Michael Burawoy, quien aporta una posible clave de interpretación sobre el sentido de reflexividad que el/la lector/a puede elegir para el recorrido sucesivo del texto. Ciencia y reflexividad, cada una, llevan el nombre de una dialéctica inherente al trabajo científico; esto es, la interacción entre teoría y datos y la interacción entre participación y observación. Ambas dialécticas comprenden, según el autor, una empresa etnográfica que cada cientista social debe afrontar sorteando las presiones que devienen del intento constante de articular sus conceptualizaciones con el dato empírico a la vez que participa, intercambia y dialoga con dos mundos: el científico -donde debe rendir cuentas a sus colegas- y el campo “exterior” -donde interactúa con sus informantes-. Frente a algunas versiones positivistas de la sociología o posmodernistas en antropología, que relativizan en cierto grado estas tensiones, el reconocimiento de esta dialéctica etnográfica se vuelve condición y promesa para una comprensión genuina de los fenómenos sociales. Más aún, subraya el autor, los tiempos para la ciencia social han cambiado, las lógicas de la mercantilización que han invadido nuestras universidades e institutos nos obligan a considerar seriamente la práctica reflexiva y su importancia en la defensa de otros espacios posibles para la producción de conocimiento.

\section{I}

En el capítulo 1, "Notas sobre la noción de reflexividad en sociología y en la obra de Bourdieu”, Denis Baranger inicia con un breve recorrido sobre los diferentes significados de la noción de reflexividad en sociología, para luego abocarse a la descripción tanto de algunos elementos contextuales que llevaron al sociólogo francés Pierre Bourdieu a emplear el término, como del sentido que adquiere en su obra y en la disciplina sociológica en general. Desde la óptica de una sociología de la sociología, Baranger reconstruye el modo en que Bourdieu fue articulando conceptualmente su preocupación epistemológica por la reflexividad a medida que confrontaba y dialogaba con diferentes disciplinas y escuelas teóricas. Este enfoque nos devuelve una mirada que no distingue diferencias fundamentales entre un concepto antropológico o sociológico de reflexividad en Bourdieu, sino que más bien emplaza su interés en el proyecto de una ciencia social reflexiva. La formulación de la idea de autoanálisis (Bourdieu, 1997) entendida como práctica reflexiva colisionó tempranamente con la epistemología popperiana, perspectiva que negaba cualquier intromisión del sujeto en el proceso de investigación social. Autoanálisis significaba dar cuenta de los condicionamientos que la posición social del/a investigador/a imprimía en su relación con el objeto de investigación: en un primer nivel, su posición en el espacio social en términos de las categorías sociales fundamentales, como clase, género y etnia; en un segundo nivel, su posición de poder dentro del campo específico de las ciencias sociales, y, por último, autoanálisis respecto de las categorías eruditas propias del campo intelectual que se habita y su relación con la práctica. 
Todo ello sin recaer en la ontologización de teorías que en todo caso deberían funcionar como caja de herramientas para desentrañar las lógicas culturales e históricas de esas prácticas e interacciones sociales que se desea investigar. Esta propuesta de autoanálisis, continúa el autor, se revelaba entonces como un socioanálisis distinto del estilo narcisista y confesional que defendía la versión reflexiva de la antropología posmoderna. Se intentaba reponer, en otro orden, elementos de una trayectoria social a partir de conceptos sociológicos que permitieran superar la esfera personal y centrar la reflexión sobre las vinculaciones posibles entre el/la investigador/a y su objeto.

Asimismo, la tarea reflexiva comportaba un segundo momento: ese autoanálisis debía ser expuesto a un examen intersubjetivo de la comunidad de expertos. Es aquí donde Baranger pone el acento en las condiciones de posibilidad que el mismo Bourdieu señalaba para este ejercicio, y lo que permite a su vez iluminar los motivos por los cuales este libro representa un aporte valioso. Si bien es en el carácter plural de sus teorías y en la multiplicidad de paradigmas donde reside la riqueza de la disciplina sociológica, ambos rasgos tienen a debilitar el grado de autonomía que todo campo disciplinar necesita para elevar el ejercicio colectivo de la práctica reflexiva. Más aún si consideramos los límites borrosos entre sociología, ciencias sociales y campo intelectual. Con atención en este diagnóstico, esta compilación contribuye al ejercicio de una reflexividad colectiva necesaria, y asume el desafío de poner en diálogo diferentes trayectorias de investigación y enfoques teórico-metodológicos desde una mirada interdisciplinar.

Desde los aportes sustanciales de la sociología bourdieuana y las controversias en torno a las posturas críticas de la antropología posmoderna la lectura del libro continúa su itinerario abriendo nuevos interrogantes en relación al concepto de reflexividad: ¿es que puede considerarse la existencia de otros aspectos relativos a la persona del/a 1 investigador/a que revistan un valor cognitivo para la investigación? ¿Qué otros significados, dimensiones o articulaciones comprenden la reflexividad? En ese sentido, otro de los abordajes complementarios y originales que acompañan el camino del lector es el capítulo siguiente, escrito por la antropóloga social y etnógrafa Rosana Guber. En “'Volando rasantes’... Etnográficamente hablando. Cuando la reflexividad de los sujetos sociales irrumpe en la reflexividad metodológica y narrativa del investigador”, la autora señala que un fundamento importante de nuestra creatividad y precisión metodológica se haya en comprender cómo los investigadores somos afectados por nuestras misiones etnográficas. El encuentro de reflexividades y la interlocución en el campo movilizan un conjunto de cargas personales, afectos, emociones y moralidades que es imprescindible restituir en la producción del dato etnográfico y en la interrogación de nuestras prácticas de investigación. De la misma manera, el grado que alcanza esa lógica intersubjetiva del conocimiento lleva a redefinir el concepto de reflexividad a partir de la contemplación y el enlace entre trabajo de campo, perspectiva teórica y elaboración del texto etnográfico. Para describir esta propuesta etnográfica sobre reflexividad, Guber vuelve sobre una crítica acerca de los modos en que académicas y académicos concebimos y enseñamos las prácticas y técnicas que empleamos, como artefactos o dispositivos universales desentendidos de sus contextos interactivos de uso y producción. Por ello propone reconstruir la experiencia de la práctica reflexiva etnográfica como "un tipo de vuelo”, recuperando su trabajo de campo junto a pilotos de la fuerza aérea que combatieron en la Guerra de Malvinas y, fundamentalmente, las formas diferenciales en que esos pilotos construían conocimientos y los daban a conocer. 
En la segunda parte del libro podremos advertir que la pregunta por la reflexividad se vuelca sobre el proceso de investigación social, las decisiones relativas a su diseño y las prácticas de investigación en sus diferentes etapas.

En el capítulo 3, primero de esta sección, titulado "Reflexividad en el proceso de investigación social: entre el diseño y la práctica”, Juan Piovani recoge un sentido de reflexividad emparentado con el análisis crítico del proceso de investigación social. Se trata de abordar dicho proceso como objeto de estudio desde una perspectiva que recupere el sentido etimológico de la metodología como revisión de las sendas o caminos que emplean las ciencias sociales para producir y validar conocimientos-. El autor reconstruye a lo largo del capítulo el conjunto de decisiones relativas al diseño que estructuran y orientan la práctica de la investigación social, y señala a su vez algunas notas reflexivas sustantivas: la necesidad de contemplar el carácter no lineal del proceso de investigación y sus etapas, la recursividad constante entre el diseño y la práctica, y la existencia de saberes tácitos y personales que deben ser explicitados y reconocidos en su valor heurístico para la investigación. Además, Piovani presenta un esquema de divisiones analíticas del proceso de investigación en tres grandes momentos o instancias, el cual resulta indispensable para comprender la organización de la exposición de los siguientes capítulos: la construcción del objeto de investigación, el trabajo de campo, y la interpretación y escritura de textos.

De este modo, la apreciación de la práctica reflexiva sobre el proceso de investigación se segmenta analíticamente de allí en adelante en esas tres instancias, y recorre diferentes enfoques de la tradición cualitativa y de métodos mixtos. Este ejercicio que propone la compilación es sumamente interesante dado que articula en un grado más íntimo y profundo la cuestión de la reflexividad, con experiencias y prácticas de investigación concretas de investigadoras e investigadores.

Comienza esta iniciativa con el enfoque biográfico, al cual se dedican los tres capítulos subsiguientes. En "Consideraciones sobre la reflexividad en el proceso de construcción de objetos de investigación biográficos”, Magdalena Lemus, Bárbara Guevara y María Eugenia Ambort tratan la problemática de la formulación de objetos de investigación, y desentrañan diversos aspectos velados en las prácticas de investigación y en la socialización de producciones escritas. En primer lugar, detallan algunos de los rasgos distintivos de la construcción de objetos biográficos. Sus preguntas parten de una dimensión temporal y de la integración de niveles que articulan subjetividades, instituciones y marcos histórico-contextuales. Seguido de ello, en diálogo con sus experiencias de investigación y las dificultades vivenciadas, las autoras relatan las operaciones reflexivas que las llevaron a reformular sus objetos iniciales en términos de sus posiciones como investigadoras, las particularidades de los sujetos de estudio y los escollos teóricos que debieron desandar en el camino.

El capítulo 5, "Hacia un encuentro de reflexividades: la entrevista biográfica como interludio del proceso de investigación social”, se aboca al análisis de la entrevista biográfica como dispositivo principal movilizado durante el trabajo de campo. Las autoras Leticia Muñiz Terra, Juliana Frassa y María de la Paz Bidauri parten de conceptualizar la entrevista como un interludio, como una composición coejecutada entre investigador y narrador situada en el momento crucial de la investigación biográfica. Esa composición condensa múltiples temporalidades y se interconecta 
integralmente con el proceso de investigación, desde la construcción del objeto hasta el análisis y la escritura de relatos de vida. En esa clave distinguen analíticamente tres momentos que atraviesan el proceso de reflexividad del narrador, antes, durante y luego del encuentro de entrevista (emergente, discursivo, resignificación), y otras tres fases reflexivas en las cuales el investigador diseña el dispositivo metodológico, participa del encuentro comunicativo y realiza un trabajo posterior de interpretación (anticipatorio, dialógico, analítico).

Por último, "Del análisis a la escritura de textos biográficos: el lugar de la reflexividad en las interpretaciones y puestas en montaje de las biografías” finaliza la exposición del enfoque biográfico en esta compilación, a cargo de Eugenia Roberti y de Leticia Muñiz Terra nuevamente. Las autoras se interesan por comprender la especificidad que asume la reflexividad del investigador en la instancia de análisis y escritura de textos biográficos y cómo a través de un trabajo centralmente interpretativo el mismo posiciona la reflexividad del sujeto narrado. En esa labor de comprensión identifican tres etapas sucesivas que comportan diferentes niveles de reflexividad para el investigador y sujeto narrado, y que consideran también el carácter que adquiere la temporalidad en cada una de ellas. Una primera etapa de registro y transcripción, en la que se intenta aprehender la reflexividad que el sujeto despliega en su relato y se recupera especialmente su temporalidad biográfica. Luego, la etapa de análisis e interpretación, en la cual se observa la presencia dialógica de ambas reflexividades y una articulación de diferentes temporalidades -biográficas, institucionales, contextuales-. Y su desenlace, en la instancia de montaje y presentación del relato biográfico, cuando adquiere primacía la operación reflexiva del investigador en la reconstrucción del relato narrado y la potenciación del ensamble de temporalidades como dispositivo de interpretación.

El capítulo 7, "Nuevas fuentes de la imaginación sociológica: la operación reflexiva y la construcción del objeto etnográfico”, da inicio a la sección etnográfica. Paula Cuestas, Martín Urtasun y Rodolfo Iuliano se detienen en el conjunto de actividades que intervienen en la construcción de objetos etnográficos para develar la eficacia y la creatividad de la operación reflexiva en la imaginación de nuevos horizontes empíricos y teóricos. Los autores interrogan lúcidamente el modo en que la consolidación de los enfoques etnográficos en las ciencias sociales vernáculas tendió a concentrar la práctica reflexiva en el encuentro e interlocución del investigador con sus "otros" informantes. Como contraparte, esta visión canónica desestimó diferentes condiciones y principios en la conformación de objetos etnográficos que los autores pretenden visibilizar y problematizar a través de un trabajo reflexivo: los consensos teóricos y recorridos bibliográficos, la filiación a diferentes tramas de circulación académica, la gravitación de las instituciones financiadoras y sus reglas, entre otros, son todos elementos que al ser objetivados permiten tomar distancia relativa de los efectos estructurantes del campo académico y propician una mirada abierta y creativa sobre las direcciones posibles de nuestras preguntas de investigación.

En el capítulo 8, "Reflexividad y roles en el trabajo de campo etnográfico”, Nicolás Aliano, Soledad Balerdi, Julia Hang y Nicolás Herrera introducen el capítulo retomando la idea de encuentro de reflexividades de Guber (2014), definición que pone el acento en el conjunto de expectativas que investigadores e interlocutores formamos unos sobre otros en los encuentros cotidianos de observación y participación. Particularmente dichos autores orientan su apuesta reflexiva sobre la 
negociación y asignación de roles que llevan adelante en sus experiencias de campo, como una herramienta analítica central para pensar la construcción del dato etnográfico. Se concentran fundamentalmente en dos ejes: en primer lugar, las expectativas que genera la presencia del investigador en el campo y los reposicionamientos que habilita; y, en segundo lugar, los procesos de “desfamiliarización/familiarización” como desafíos que se asumen a lo largo del trabajo de campo en pleno diálogo con las reflexividades propias de nuestros interlocutores.

Al cierre del eje etnográfico encontramos “¿Un pase de magia? Ejercicios de reflexividad a través de dos procesos de análisis etnográficos”, escrito por Ornela Boix y Nicolás Welschinger. Ambos autores recorren otras de las zonas oscuras para el ejercicio de la reflexividad metodológica en la etnografía, aquella que repara específicamente en el momento de la interpretación y análisis de datos, y la producción textual. Sin recaer en un examen abstracto de las técnicas y sus resultados, eligen dialogar con la literatura especializada a partir de un proceso de concretización de las prácticas, es decir, de fomentar dos procesos de análisis reflexivo sobre la base de sus etnografías en curso. Así, los autores sugieren que para sortear los polos de riesgo por los cuales circula cualquier análisis etnográfico -el empirismo ingenuo y la sobreinterpretación teoricista- se debe producir un descentramiento del investigador que promueva una interlocución triple: entre su propia reflexividad, la comunidad erudita con la que dialoga y sus informantes. En ese tránsito de reflexividades es que se puede “arriesgar la interpretación”: una propuesta de análisis atractiva que articula la apuesta por reposicionar la tarea de descripción en sociología -desde una formulación crítica al positivismo-, y una concepción de la escritura como mediación productiva del análisis mismo.

Finalmente, el libro concluye con una última ambición, la de llevar la interpelación reflexiva fuera de la tradición cualitativa "haciendo pie" sobre los enfoques metodológicos mixtos. Javier Santos, Pilar Pi Puig y María Eugenia Rausky asumen esa tarea en el capítulo 10 titulado "Métodos mixtos y reflexividad: explorando posibles articulaciones”. Los autores parten del reconocimiento del escaso abordaje de la cuestión reflexiva en las producciones científicas basadas en métodos mixtos. Para introducir esta pregunta comienzan primero describiendo algunas de las características centrales de los métodos mixtos, para luego evaluar la presencia, usos y condiciones de posibilidad de la práctica reflexiva en los mismos. Se basan para ello en la revisión de la Journal of Mixed Methods Research (JMMR), primera publicación periódica internacional que se focaliza en la difusión de artículos empíricos, metodológicos y teóricos asociados a este enfoque. Como corolario, ofrecen dos pistas por las cuales seguir pensando el vínculo entre reflexividad y métodos mixtos. Una dimensión teórica, que problematice los modos en que podemos dar cuenta de las mediaciones entre lo subjetivo y objetivo; y una segunda dimensión epistemológica, que se preocupe por las relaciones entre el punto de vista externo del investigador y los sujetos implicados, y su incidencia en las decisiones relativas al diseño e implementación. Esta última dimensión reflexiva puede resultar especialmente fructífera, dado que puede propiciar la emergencia de perspectivas innovadoras que colaboren con nuevas formas de validación de conocimiento asociadas a la utilización de métodos mixtos. 


\section{Notas}

1 Esta reseña adhiere al uso de un lenguaje inclusivo en términos de género, pero para facilitar la lectura se utilizará el universal masculino.

\section{Referencias bibliográficas}

Bourdieu, P. (1997). Méditations pascaliennes. París: Seuil.

Guber, R. (2014). La etnografía: Método, campo y reflexividad. Buenos Aires: Siglo Veintiuno Editores. 